\title{
Research article \\ Gait parameters and functional performance following multi-factorial treatment among degenerative joint disease patients
}

\author{
A. D. Gopalswami ${ }^{1}$, S. Senthil Kumar ${ }^{2}$, A. G. Sinha ${ }^{3}$ \\ ${ }^{\mathbf{1}}$ Associate Professor, Faculty of Physiotherapy, ${ }^{\mathbf{2}}$ Professor of Anatomy and Principal AHS, Sri Ramachandra Institute of \\ Higher Education and Research (Deemed to be University), Porur, Chennai 600116, Tamil Nadu, India \\ ${ }^{3} \mathrm{Head}$, Department of Physiotherapy, Punjabi University, Patiala, Punjab, India
}

(Received: September $2020 \quad$ Revised: February $2021 \quad$ Accepted: March 2021)

Corresponding author: S. Senthil Kumar. Email: ssksrmc@gmail.com

\begin{abstract}
Introduction and Aim: Degenerative Joint Disease (DJD) poses a challenge to manage and restore functional capabilities among patients. Incorporation of structured exercises, manual therapy and patient education had a significant impact on clinical and functional status of patients. Past research has explored benefits of therapy on functions and gait. Although, such studies did not address the functional restoration. Hence, present study was aimed to address the lacunae.
\end{abstract}

Materials and Methods: 100 patients with mean age and SD; 51.61(5.4) were included. Participants had knee Degenerative Joint Disease (DJD); grade 2 or 3 on Kellgren and Lawrence scale, were allocated to Experimental Group (EG) and Comparative Group (CG). EG received joint mobilization and Muscle Energy Technique (MET). CG received Interferential therapy (carrier frequency $4 \mathrm{kHz}$; beat frequency $100 \mathrm{~Hz}$; and sweep frequency $150 \mathrm{~Hz}$ ). Strengthening exercises and patient education were common for both groups. Intervention lasted two weeks, the postintervention metrics included; stride length, step length, and cadence for gait and knee injury and osteoarthritis outcome score.

Results: Data obtained was subjected to statistical analysis. Frequency and percentage were used for categorical variables; mean and standard deviation (SD) were used for continuous variables. Post-intervention, there was a significant improvement of gait parameters and Knee Injury and Osteoarthritis Outcome Score (KOOS) in both groups. CG had a better improvement in STL, SL, CAD with mean of 39.84(5.68), 20.22(2.84), 97.16(3.75) and EG with mean of 44.36(4.48), 22.30(3.75), 92.82(3.75) and $p<.0005$. However, it was evident that the proportion of functional improvement among CG was lesser.

Conclusion: Inclusion of structured exercises, manual therapy and patient education in intervention would be effective and improve the functional status of DJD patients.

Keywords: MET; exercises; manual therapy; functional performance; degenerative joint disease.

\section{INTRODUCTION}

$\mathrm{K}$ nee is a weight-bearing joint of lower limb, which primarily functions to lengthen and shorten the lower extremity. This function assists hip in positioning the foot for various functional activities and gait. Degenerative Joint Disease (DJD) is a musculoskeletal disorder that affects a large proportion of population throughout the world. It predominantly affects the weight bearing joints such as hip, knee, and vertebral column. The prevalence of DJD is more common among Indian than western population in relation to lifestyle pattern, primarily targeting medial and lateral tibiofemoral compartment of knee than the patellofemoral compartment (1).

The DJD may have a varied clinical manifestation. Some of the common presentations may include pain that increases with activities, swelling around the joint, warmth, and crepitus during movement and stiffness in the knee joint. In addition, shortening of hamstring muscles is common among patients with
DJD, which poses a significant problem with normal gait and Activities of Daily Living (ADL). DJD has multiple choices of therapeutic interventions. It includes medical management, and forms of Physiotherapy which includes pain management, manual therapy, exercises in order to enhance joint mobility, muscle efficiency and activity modification etc., (2). The primary focus of physiotherapy during later stages of disease may include means to improve stability, range of motion, gait and patient education.

Reduced flexibility of skeletal muscles has been managed by many methods in Physiotherapy. Exercise plays a vital role in improving the flexibility and strength of knee musculature. Puhakka et al., stated that, clinically Muscle Energy Technique (MET) proves to be an effective method in alleviating tightness and very little research was conducted in the past using MET as a part of treatment. MET works based on Gate Control Theory, which is one mechanism for altered stretch tolerance and pain perception as stated by Melzack and Wall. Kuchera and Kuchera explained post-isometric relaxation 
technique and it creates muscle relaxation that occurs after applying isometric contraction of the muscle (3).

Muscle loss significantly contributes to the disability and morbidity associated with DJD of knee and thigh muscle weakness is an independent, modifiable risk factor (4). While treatment guidelines recommend strengthening, exercise to combat weakness in patients with DJD of knee one of the most important finding was the general deficit in muscular strength along the affected limb as compared to the contralateral (healthy) side in patients with unilateral knee DJD. In addition, the related muscles showed marked atrophy as evidenced by reduced cross sectional area as well as maximal voluntary isometric contraction (5). Therefore, mentioned findings may greatly affect gait and functional capability of an individual with DJD of knee.

There has been a report on therapeutic benefits of exercise on DJD symptoms. It not only reduces symptoms but also delays disease progression and is thus beneficial to patients. Quadriceps muscle weakness is a frequent finding among patients with knee DJD and is associated with pain intensity, physical dysfunction, and decreased function. Quadriceps strengthening is commonly prescribed for DJD of knees because it reduces pain and improves function (6).

Impairment of walking ability is one of the most important determinants of disability. It is manifested as a decrease in gait speed and cadence, whereas gait cycle duration and double-limb support time increases due to shorter stance phase. Gait deficit may contribute to increased risk of falls. Therefore, gait recovery may improve overall functioning and wellbeing, and it represents a key goal in the management of DJD patients (7).

Gait analysis is important to evaluate walking function. Its analysis using instrumentation has been accepted as the gold standard for the evaluation of this function, as it provides reliable and accurate information in the three planes movement (8). However, these techniques have disadvantages of high economical cost and difficult access as they are not available for all professionals, the instrumented analysis is complex, requires time resources, and high level of skill in its use (9). These disadvantages of instrumented gait analysis have led to the development of a variety of observational analysis, which is more viable than instrumented analysis (10). Also, it has low cost and does not require specialized equipment or location. Such analysis has been recommended for patients with orthopedic disorders (11). Thus, Observational gait assessment have become an effective alternative due to its ease of use, probably becoming the most commonly used method in clinical set up.
The Knee Injury and Osteoarthritis Outcome Score (KOOS) is a knee-specific instrument, developed to assess the patients' opinion about their knee and associated problems. It is a valid instrument to measure the clinical condition of patients undergoing treatment of a focal cartilage lesion (12).

Thus, the physical impairments among DJD patients poses a significant challenge to clinicians in order to manage and restore their functional capabilities. The potential benefits of conventional exercise program lasted for short term and a possible recurrence of symptoms, whereas incorporation of structured exercises, manual therapy and patient education had a significant positive impact on clinical and functional status of the patients. There has been quite a number of researchers in the past that have attempted to find the benefits of exercises and manual therapy on improving gait (13). However, very few studies have correlated them as means of treatment in finding the efficacy for DJD patients. Although past research identified the most effective non-operative methods of treatment, the intervention in such studies did not address the issue of functional restoration of the patient. Thus, present study was attempted to address the muscular deficits viz, flexibility and strength and hence functional restoration. The primary aim was to find out the efficacy of multifactorial treatment such as MET, joint mobilizations and structured exercise program on patients with DJD of knee and the objectives of the study were

1. To perform an observational analysis of phases of gait cycle.

2. To analyze the effectiveness of manual therapy (MET and joint mobilizations), exercise program in improving the gait parameters

3. To determine the functional performance utilizing Knee Injury and Osteoarthritis Outcome Score (KOOS).

\section{MATERIALS AND METHODS}

This prospective comparative study comprising of one hundred subjects was conducted in Outpatient Physiotherapy Department of Sri Ramachandra Hospital, Chennai, India. Institutional Ethics Committee, IEC approval number (CSP/12/SEP/25/130) prior to commencement, approved the study and a written informed consent was obtained from all the participants.

The estimated sample size for study was calculated using actual data on efficacy of two different intervention programs conducted by Aline Mizusaki Imoto de Oliveira. Using a two-sample comparison of means needed at least 52 patients in each group. The sample size was estimated to be 104 samples (52 in each group). There were four-dropouts out of which three subjects refused to continue after few sessions quoting their unwillingness and one of subject could 
not be included till the end as they developed other medical issues. Thus, the sample size was calculated and rounded to 100 (50 in each group). The sample size was arrived with $\mathrm{n}$ Master software Version 2.0 by applying the formula for hypothesis testing for two means.

\section{Inclusion criteria}

Patients diagnosed with Unilateral DJD of knee, both gender with age interval of 40 to 60 years. Knee pain for 6 months or more that was unresponsive to (1) conservative treatments - oral analgesics (2) Visual Analogue Scale (VAS) pain score of 6 or greater for the knee; (3) radiological confirmation of OA - mildto moderate radiographic tibiofemoral DJD (KellgrenLawrence $(\mathrm{KL})=2$ or 3 ).

\section{Exclusion criteria}

Patients with patello-femoral pain syndrome, severe valgus/varus deformity of the knee, past or recent fracture around the knee region, active infections in the knee joint, post traumatic arthritis, knee instability secondary to ligament injuries, past surgeries in the knee and limb length discrepancy were excluded.

Those who met inclusion criteria had participated in the study and were explained about their clinical condition, details of the study and potential benefits of participation. Random numbers allocated the study participants to the Experimental Group (EG) and the Comparison group (CG) by a concealed method in order to eliminate selection bias and confounding variables.

Baseline measures of gait variables such as Stride Length (STL), Step Length (SL) and Cadence (CAD) was done using 20-meter walk test. During the observational analysis of phases of gait cycle, subjects were asked to walk at a self-selected comfortable speed along a line of progression for a distance of 20 meters. It was observationally assessed using the footprint method. The metrics were taken during initial evaluation and following intervention, with an interval of two weeks. Stride length was measured as the distance from heel contact mark to heel contact mark by the same foot and was calculated by averaging the strides. The step length measurement was linear distance of an average of the steps on one side of the line of progression from the left foot contact to the right foot contact. Cadence was calculated as number steps taken per minute using a stopwatch (14).

The functional status was evaluated using KOOS. It evaluates both short-term and long-term consequences of knee injury and degeneration. The tool holds 42 items in five separately scored subscales: Pain, other Symptoms, Function in daily living (ADL), Function in Sport and Recreation (Sport/Rec), and knee-related Quality of Life (QOL). This is self-administered, proved reliable, responsive to surgery and physical therapy. It meets basic criteria of outcome measures and is widely used to evaluate the course of knee injury and treatment outcomes. Average completion time for KOOS ranged from 7 to 12 minutes and manual data entry and scoring took $10 \mathrm{~min}$ (15). Qualified Physiotherapists did the initial and periodical evaluations of all patients.

The intervention consisted of physiotherapy sessions including manual therapy, mobility/strengthening exercises, pain relieving modalities and patient education. The patients belonging to EG had undergone an intervention, which included manual therapy (joint distraction and mobilization), MET, strengthening exercises and patient education whereas those belonging to the CG had undergone the referred treatment program (Interferential Therapy and exercises) along with strengthening exercises and patient education. Interferential therapy had parameters for pain modulation with carrier frequency $4 \mathrm{kHz}$; beat frequency $100 \mathrm{~Hz}$; and sweep frequency $150 \mathrm{~Hz}$, using quadripolar technique (16). The program lasted for two weeks with an average frequency of three sessions per week and duration of 30 min each session once a day for both the groups at the physical rehabilitation outpatient department of Sri Ramachandra Hospital, Chennai.

\section{Intervention}

\section{Manual distraction}

The patient was positioned in high sitting with thigh stabilized by a belt strapped to the couch. Manual distraction was applied by grasping the distal end of lower limb above the malleoli and held for 7 to 10 counts.

\section{Tibio-femoral glides}

Antero-Posterior (AP) glides were performed with the patient supine and lower limp propped, reaching maximal extension. The proximal tibia is being stabilized with one hand and the mobilizing hand placed on the distal femur and a posteriorly directed force was applied directly downward through the distal femur. For Postero-Anterior (PA) glides, the patient was in supine with the knee in mid flexed position the mobilizing hands placed just below the tibial tuberosity. The glide was performed by a force perpendicular to the line of tibia. Both the glides were performed for 7 to 10 oscillations in each direction.

\section{Muscle Energy Techniques (MET)}

The MET procedures as described by Chaitow were used in the present study. The techniques included, Post Isometric Relaxation (PIR) and Reciprocal Inhibition (RI), performed to improve the flexibility of hamstrings and thereby improve knee extension (17).

\section{Exercises}

Both the groups had undergone an exercise program based on patient's ability and within pain free range. 
It included therapeutic open kinematic chain and strengthening exercises. For strengthening exercises, 1RM (Repetition Max) was defined as the maximal resistance one can lift in a single repetition. 1RM tests determine the starting resistance used for each exercise in the subsequent sessions. Participants had maintained a session log of each resistance exercise, its weight setting, and number of sets and repetitions achieved. The exercises include:

1. Maximal static quadriceps contraction (quadriceps setting) with knee in extension.

2. Short arc movements from knee flexion of desired angle to terminal knee extension.

3. Straight leg raising with patient in supine.

4. Leg curl and extension.

5. Hip abduction, adduction and extension.

\section{Patient education}

Participants belonging to both groups had a common patient education program. The patient education was based on Arthritis Self-Management Program (ASMP), developed by a team of researchers at Stanford University. This was designed for patients with arthritis adapt to their condition and an improved quality of life (18). It included components like home exercise program, joint protection principles, measures to maintain balance and prevent fall, selfmanagement and lifestyle modification.

\section{Statistical analysis}

The data collected was analysed with IBM, SPSS statistics software 23.0 version. In order to describe the data descriptive statistics, frequency analysis, percentage analysis was used for categorical variables and the mean and Standard deviation (SD) were used for continuous variables. Various choice of statistical tests was used based on the type of variables. The significant difference between the bivariate samples in paired groups was analysed using paired sample t-test \& the Wilcoxon signed rank test. As far as the Independent group analyses, the Unpaired sample ttest and the Mann-Whitney $\mathrm{U}$ test were used. The significance in categorical data was found using Chi-
Square test. In all of the above statistical tools, the probability value of $p \leq 0.05$ was considered as level of significance.

\section{RESULTS}

The group statistics for age has been depicted in Table 1 , which reveals that equal variances have been assumed to an extent. Wilcoxon signed rank test was used to analyze outcome variables for gait (STL, SL and $\mathrm{CAD}$ ) within the groups (Table 2). Following intervention, there has been a significant improvement in gait variables (STL, SL and CAD) among both the groups $(\mathrm{p}=.005)$. Although, the $\mathrm{CG}$ had a better improvement in gait variable STL, SL, CAD with a mean of 39.8(5.7) inches, 20.2(2.8) inches, 97.1(3.7) steps against the EG with a mean of 44.3(4.5) inches, 22.30(3.7) inches, 92.8(3.7) steps (Table 3).

The post intervention functional status was evaluated using KOOS had significantly improved among both the groups. The KOOS subdomain ssymptoms (SYM), pain (PAIN), activities of daily life (ADL), function in sports and recreation (FXN), knee related quality of life (QOL) \} scores were also individually analyzed. EG had better improvement in KOOS subdomains mean of SYM 90.10(5.93), PAIN 82.22 (7.51), ADL 81.96(6.44), FXN 69.70(6.50), and QOL 81.20 (3.28) as compared to CG KOOS subdomains mean of SYM 78.92(4.62), PAIN 85.70(8.00), ADL 78.60(3.97), FXN 62.50(3.39), and QOL 73.90 (5.08) (Table 2). Upon analysis of KOOS score, it was evident that the proportion of functional improvement among the CG was lesser.

Mann Whitney U test was used to compare the post intervention outcomes between the groups. The gait variables had a better improvement among the experimental than the comparison group. In addition, it was found to be highly significant statistically, \{ STL $(\mathrm{p}=.005)$, SL ( $\mathrm{p}=.004$, CAD ( $\mathrm{p}=.008)$. Likewise, KOOS subdomain scores had also improved better among the experimental than the comparison group, SYM ( $\mathrm{p}=.005)$, PAIN $(\mathrm{p}=.025)$, ADL $(\mathrm{p}=.007)$, FXN $(\mathrm{p}=.005)$, QOL $(\mathrm{p}=.005)($ Table 3$)$.

Table 1: Demographics

\begin{tabular}{|c|c|c|c|}
\hline & \multicolumn{2}{|c|}{ Group } & \multirow[b]{2}{*}{ p value } \\
\hline & EG & CG & \\
\hline Gender (Male/Female) & $50(17 / 33)$ & $50(14 / 36)$ & \multirow[b]{2}{*}{.956} \\
\hline Age - Mean(SD) & $51.64(5.29)$ & $51.58(5.63)$ & \\
\hline 45 years & 8 & 10 & \\
\hline $46-50$ years & 11 & 8 & \\
\hline $51-55$ years & 22 & 21 & \\
\hline \multirow[t]{2}{*}{$56-61$ years } & 9 & 11 & \\
\hline & \multicolumn{2}{|c|}{ Mean (SD) } & \\
\hline Height $(\mathrm{cm})$ & $158.71(6.22)$ & $165.59(2.55)$ & \\
\hline Weight (Kg) & $60.3(4.95)$ & $63.84(5.62)$ & \\
\hline BMI & $28.1(2.82)$ & $23.29(2.05)$ & \\
\hline
\end{tabular}

Table 2: Comparison of variables within groups 


\begin{tabular}{|c|c|c|c|c|c|}
\hline \multicolumn{2}{|c|}{ Variables/ Group } & Pre intervention & Post intervention & $\begin{array}{c}\text { Mean } \\
\text { difference }\end{array}$ & p value \\
\hline \multicolumn{3}{|c|}{ Gait } & \multicolumn{3}{|c|}{\begin{tabular}{l|l} 
& difference \\
Mean(SD)
\end{tabular}} \\
\hline \multirow{2}{*}{$\begin{array}{c}\text { Stride Length } \\
\text { (Inches) }\end{array}$} & EG & $19.70(1.11)$ & $44.36(4.48)$ & $24.66(4.36)$ & $.0005^{*}$ \\
\hline & $\mathrm{CG}$ & 19.94(1.33) & $39.84(5.68)$ & $19.90(5.57)$ & $.0005^{*}$ \\
\hline \multirow{2}{*}{$\begin{array}{c}\text { Step Length } \\
\text { (Inches) }\end{array}$} & EG & $14.60(1.13)$ & $22.30(3.75)$ & $7.70(3.69)$ & $.0005^{*}$ \\
\hline & $\mathrm{CG}$ & $14.48(1.07)$ & $20.22(2.84)$ & $5.74(3.00)$ & $.0005^{*}$ \\
\hline \multirow{2}{*}{$\begin{array}{c}\text { CAD } \\
\text { (Steps/minute) }\end{array}$} & EG & $82.90(4.45)$ & $92.82(3.75)$ & $9.92(3.96)$ & $.0005 *$ \\
\hline & $\mathrm{CG}$ & $90.14(5.28)$ & $97.16(3.78)$ & $7.020(6.47)$ & $.0005 *$ \\
\hline \multicolumn{6}{|c|}{ KOOS } \\
\hline \multirow[b]{2}{*}{ Symptoms } & EG & $47.92(5.58)$ & $90.10(5.93)$ & $42.18(2.67)$ & $.0005^{*}$ \\
\hline & $\mathrm{CG}$ & $46.44(6.98)$ & $78.92(4.62)$ & $32.48(5.09)$ & $.0005^{*}$ \\
\hline \multirow[b]{2}{*}{ Pain } & EG & $41.54(7.31)$ & $82.22(7.51)$ & $40.68(4.17)$ & $.0005^{*}$ \\
\hline & $\mathrm{CG}$ & $41.14(7.81)$ & $85.70(8.00)$ & $44.56(11.35)$ & $.0005 *$ \\
\hline \multirow{2}{*}{$\begin{array}{c}\text { Activities of } \\
\text { daily life }\end{array}$} & EG & $41.30(5.64)$ & $81.96(6.44)$ & $40.66(2.78)$ & $.0005^{*}$ \\
\hline & $\mathrm{CG}$ & $40.90(6.07)$ & $78.60(3.97)$ & $37.70(7.05)$ & $.0005^{*}$ \\
\hline \multirow[b]{2}{*}{ Function } & EG & $11.40(6.23)$ & $69.70(6.50)$ & $58.30(3.29)$ & $.0005^{*}$ \\
\hline & $\mathrm{CG}$ & $11.00(5.62)$ & $62.50(3.39)$ & $51.50(3.95)$ & $.0005^{*}$ \\
\hline \multirow[b]{2}{*}{ Quality of Life } & EG & $30.00(6.35)$ & $81.20(3.28)$ & $51.20(4.46)$ & $.0005^{*}$ \\
\hline & $\mathrm{CG}$ & $26.50(2.64)$ & $73.90(5.08)$ & $47.40(3.87)$ & $.0005^{*}$ \\
\hline
\end{tabular}

Note: To find the significant difference between the bivariate samples in paired groups the paired sample t- test and Wilcoxon signed rank test were used. To find the significance in categorical data Chi-Square test was used.

EG: Experimental Group, CG: Comparison Group.

STL - Stride length (Units - inches), SL - Step length (Units - inches), CAD - Cadence (Units - Steps per minute), KOOS - Knee Osteoarthritis Outcome Score, SYM - Symptoms, ADL - Activities of daily life, FXN: Function, sports and recreation, QOL - Knee related quality of life.

$\mathrm{t}$ test $* \mathrm{p}$ value $<.05$ significant.

Table 3: Comparison of variables between the groups

\begin{tabular}{|c|c|c|c|c|}
\hline Gait & EG & CG & $\begin{array}{c}\text { Between group } \\
\text { difference }\end{array}$ & p value \\
\hline $\begin{array}{c}\text { Stride length } \\
\text { (Inches) }\end{array}$ & $44.4(4.5)$ & $39.8(5.7)$ & $4.76(1.00)$ & $.005^{*}$ \\
\hline $\begin{array}{c}\text { Step Length } \\
\text { (Inches) }\end{array}$ & $22.3(3.7)$ & $20.2(2.8)$ & $1.96(0.67)$ & $.004^{*}$ \\
\hline $\begin{array}{c}\text { CAD } \\
\text { (Steps/minute) }\end{array}$ & $92.8(3.7)$ & $97.1(3.8)$ & $2.90(1.07)$ & $.008^{*}$ \\
\hline \multicolumn{5}{|c|}{ KOOS } \\
\hline Symptoms & $90.10(5.93)$ & $78.92(4.62)$ & $9.70(0.81)$ & $.000^{*}$ \\
\hline Pain & $82.22(7.51)$ & $85.70(8.00)$ & $-3.88(1.71)$ & $.025^{*}$ \\
\hline $\begin{array}{c}\text { Activities of } \\
\text { daily life }\end{array}$ & $81.96(6.44)$ & $78.60(3.97)$ & $2.96(1.07)$ & $.007^{*}$ \\
\hline Function & $69.70(6.50)$ & $62.50(3.39)$ & $6.80(0.73)$ & $.0005^{*}$ \\
\hline Quality of Life & $81.20(3.28)$ & $73.90(5.08)$ & $3.80(0.84)$ & $.0005^{*}$ \\
\hline
\end{tabular}

Note: To find the significant difference between the bivariate samples in Independent groups the Unpaired sample t-test and the Mann-Whitney U test were used. To find the significance in categorical data Chi-Square test was used.

EG: Experimental Group, CG: Comparison Group

STL - Stride length (Units - inches), SL - Step length (Units - inches), CAD - Cadence (Units - Steps per minute), KOOS - Knee Osteoarthritis Outcome Score, SYM - Symptoms, ADL - Activities of daily life, FXN - Function, sports and recreation, QOL - Knee related quality of life.

Unpaired $t$ test $* p$ value $<.05$ significant.

\section{DISCUSSION}

The tibio-femoral joint sustains large stresses during functional activities and normal gait. Quadriceps and Hamstrings are the muscles that contributes to stability of knee and are active during normal gait, decelerate the extension of knee in late swing and to help extend hip in stance phase. Muscle imbalance causes abnormal stress on the joint and can lead to joint degeneration. Co-contraction of hamstring and quadriceps helps to stabilize knee and protect the surrounding ligamentous structures. It is necessary clinically, to recognize the impact of weakness, gait 
disturbances and impaired functions among patients suffering degenerative joint disease. Thus, the main purpose of study was to find out the efficacy of multifactorial treatment such as MET, joint mobilizations and structured exercise program on patients with DJD of knee.

The results illustrate that there was significant improvement in gait variables (STL, SL \& CAD) and functional status (KOOS) among both the groups with subtle variations.

As far as the pain intensity was concerned, there was a significant reduction of pain among both the groups, although a greater proportion of pain reduction was among the EG. Such an outcome relates to the shortterm pain relief for DJD of knee with the use of interferential currents for pain modulation (19). Although, the administration of MET and tibio femoral joint glides led to the pain relief among the experimental group. These methods are known for its immediate relief of pain following administration for a single session, and this effect is not impermanent because it has no effect on reducing the severity of structural changes, especially within cartilage (20).

The ROM of knee extension had improved both clinically and statistically with significance. The reason attributable was the application of joint mobilization techniques, which included distraction, AP and PA glides to tibio-femoral joint. We could correlate a similar outcome, which concluded that there has been convincing evidence for the effectiveness of manual therapy for knee joint ROM improvement. In addition, manual therapy was considered superior to mere exercise program in benefitting the patients by and large (21). This recent evidence also favors the outcome of present study findings.

MET was chosen over passive stretching in improving flexibility of hamstrings and thereby increasing the knee joint extension during gait and functional activities. There has been adequate support for the efficacy of MET which indicated that muscle energy technique, PNF stretching and static stretching produce a significant improvement in hamstring flexibility and hence may be used as an effective therapeutic maneuver for decrease pain, improving ROM and increase flexibility of tight hamstring (22).

Few researchers have reported the usefulness of MET in the treatment of trigger points, hypomobile joints, and weak muscles. Wilson et al., concluded that MET combined with supervised motor control and resistance exercises might be effective for improving strength and ROM thereby decreasing disability. Thus, a combination of MET and strengthening exercise program was adopted with an aim to address the strength deficits of knee musculature in DJD patients. The Post Isometric Relaxation (PIR) and Reciprocal Inhibition (RI) techniques were used. The
Hamstrings and Quadriceps muscles were recruited to contract isometrically with a submaximal resistance maintained for 7 seconds as described by Chaitow (17).

The strength relationship between the quadriceps and hamstring muscles has been and reported by past studies. The ratio of the quadriceps to hamstring muscle strength is important for the stability of the knee as well as protection from excessive stress. Strengthening of the hamstring muscle has been found to enhance the functional ability of a deficient knee. The strengthening program was adopted from a previous recommendation for patients with DJD of knee (23). Biomechanically, it is clear that large external knee flexion moments require large knee extensor muscle torque to prevent collapse of the lower extremity during gait (24).

Evaluation of the treatment effect revealed that, patients belonging to EG showed specific gait adaptations, including an increased STL, SL and CAD. This change is attributable to specific therapy administered to improve flexibility of hamstrings, strengthening of quadriceps. Significantly, lower scores of physical functions as well as decreased pain in KOOS suggested that patients with knee DJD responded favorably to the exercise program used in the current study. The significantly improved physical function displayed in the current study is consistent with the findings of a past research of Chang et al., (13). Aforementioned results and our finding of the improved gait parameters confirm the positive effect of exercise on walking efficiency.

The KOOS is a knee-specific instrument, developed to assess the patients' opinion about their knee and associated problems. The KOOS questionnaire was developed in the 1990s as an instrument to assess the patient's opinion about their knee and associated problems. Inclusion of two different subscales of physical function relating to daily life, and sport and recreation. This enhances the instrument's validity for patients with a wide range of current and expected physical activity levels. Assessment changes from week to week induced by treatment (medication, operation, physical therapy) or over years following a primary injury or DJD.

The psychometric properties of the KOOS have been assessed in more than twenty individual studies from all over the world. Thus, KOOS demonstrates adequate content validity, internal consistency, test-retest reliability, construct validity and responsiveness for age and condition-relevant subscales. The KOOS has consistently shown acceptable responsiveness for different populations Suggested order of subscales for different knee conditions can be applied in hierarchical testing of endpoints in clinical trials. (12). 
The KOOS scores had significantly improved following intervention for both the groups. The analysis of KOOS scores revealed that the overall functional status had significantly improved among both EG and CG. Further analysis of the subdomains of KOOS showed that Symptoms, ADL, Functions in Sports and recreation, and QOL had a better score except for pain. The better pain reduction among CG may be attributable to the treatments and modalities used for pain modulation. Although the proportion of difference was very minimal. Such findings correlate to past studies focusing on its immediate relief of pain following administration for a single session (19).

Evidence has indicated that the level of patient selfreliance required to sustain the exercise program through a home-based program determined the exercise effect. Hence, in this study, monitoring sheets were used for the exercise program to ensure a sufficient level of patient self-reliance to sustain the improvements, which may have been another contributor to the identified clinical improvement.

\section{Limitations}

Gait was the one of the primary outcomes considered, which was performed by observational analysis. If the same could have been done in real time when assessing subjects with three-dimensional gait analysis outcomes would have been more accurate. In addition, a cross sectional analysis of similar outcomes as a longitudinal study may be considered.

\section{CONCLUSION}

The experimental group had received manual therapy including tibio-femoral glides, MET and structured exercise program. The comparison group received standardized exercise therapy and pain modality. The outcome measures were chosen to reflect positive changes in gait parameters and functional status (KOOS) compared with those participants managed with conventional treatment. There is evidence to claim that inclusion of manual therapy as a part of treatment regimen would be a comprehensive management for patients with DJD of knee. In addition, results of the study will provide evidence to guide clinicians to establish priorities regarding the provision of disease specific program in order to improve the gait and functional performance. Such a conservative treatment of DJD may reduce the number of patients interested in having surgery and can possibly delay or reduce the need for surgical joint replacement. Future studies may focus using technical advancements in order to analyze the gait parameters. In addition, the functional outcome may be compared using different disease specific measures for knee DJD.

\section{ACKNOWLEDGEMENT}

I wish to thank all the people whose assistance was a milestone in completion of this project. My special gratitude to Dr. Seppan Prakash, Associate Professor, Department of Anatomy, Dr. ALM PGIMS, University of Madras, Taramani, Chennai. Prof. N Venkatesh, Chairman, Faculty of Physiotherapy, SRIHER. Prof. R Sivakumar, Principal, Faculty of Physiotherapy, SRIHER, Prof. Antony Leo Aseer, Vice principal, Faculty of Physiotherapy, SRIHER. Prof. Narasimman Swaminathan, Vice Principal, FOAHS, Sri Ramachandra Institute of Higher Education and Research, Porur, Chennai.

\section{CONFLICT OF INTEREST}

Authors state no conflict of interest.

\section{REFERENCES}

1. Plotnikoff, R., Karunamuni, N., Lytvyak, E., Penfold, C., Schopflocher, D., Imayama, I., et al., Osteoarthritis prevalence and modifiable factors: a population study. BMC Public Health. 2015 Dec; 15(1): 1195.

2. Grazina, R., Andrade, R., Bastos. R., Costa, D., Pereira, R., Marinhas, J., et al., Clinical Management in Early OA, A dv Exp Med Biol. 2018; 1059: 111-135.

3. Ballantyne, F., Fryer, G., McLaughlin, P. The effect of muscle energy technique on hamstring extensibility: the mechanism of altered flexibility. Journal of osteopathic medicine. 2003 Oct 1; 6(2): 59-63.

4. Slemenda, C., Brandt, K. D., Heilman, D. K., Mazzuca, S., Braunstein, E. M., Katz, B. P., et al., Quadriceps weakness and osteoarthritis of the knee. Ann Intern Med. 2016; 127: 97-104.

5. Rasch, A., Byström, A. H., Dalen, N., Berg, H. E. Reduced muscle radiological density, cross-sectional area, and strength of major hip and knee muscles in 22 patients with hip osteoarthritis, Acta Orthopaedica. 2009; 78(4): 505-510.

6. Bennell, K. L., Kyriakides, M., Metcalf, B., Egerton, T., Wrigley, T. V., Hodges, P. W, et al., Neuromuscular versus quadriceps strengthening exercise in patients with medial knee osteoarthritis and varus malalignment: a randomized controlled trial. Arthritis \& Rheumatology. 2014 Apr; 66(4): 950-959.

7. Toro, B., Nester, C., Farren, P. A review of observational gait assessment in clinical practice. Physiother Theory Pract. 2003; 19: 137-149.

8. Bella, G. P., Rodrigues, N. B., Valenciano, P. J, Silva, L. M., Souza, R. C. Correlation among the visual gait assessment scale, Edinburgh visual gait scale, Brazilian Journal of Physical Therapy. 2012 Apr; 16(2): 134-140.

9. Bryant, M. S., Rintala, D. H., Hou, J. G., Protas, E. J. Reliability of the non-instrumented walk test in persons with Parkinson's disease. Disabil Rehabil. 2013; 35(7): 538-542.

10. Orozco, M. D., Abousamra, O., Church, C., Lennon, N., Henley, J., Rogers, K.J., et al., Reliability and validity of Edinburgh visual gait score as an evaluation tool for children with cerebral palsy. Gait \& posture. 2016 Sep 1; 49: 14-18.

11. Macri, F., Marques, L. F., Backer, R. C., Santos, M. J., Belangero, W. D. Validation of a standardized gait score to predict the healing of tibial fractures. The Journal of bone and joint surgery. British volume. 2012 Apr; 94(4): 544-548.

12. Collins, N. J., Prinsen, C. A., Christensen, R., Bartels, E. M., Terwee, C. B., Roos, E. M et al., Knee injury and osteoarthritis outcome score (KOOS): systematic review and meta-analysis of measurement properties. Osteoarthritis and Cartilage. 2016 Aug 1; 24(8): 1317-1329.

13. Chang, S. Y., Lin, Y. J., Hsu, W. C., Hsieh, L. F., Lin, Y. H., Chang, C. C., et al., Exercise alters gait pattern but not knee load in patients with knee osteoarthritis. BioMed research international. 2016; Article ID 7468937.

14. Faqih, A., Gavankar, U., Tambekar, N., Rairikar, S., Shyam, A., Sancheti, P., et al., Effect of rigid taping on pain and gait 
parameters in knee osteoarthritis. International Journal of Current Research and Review. 2015 Jan 1; 7(1): 24-27.

15. Roos. E. M., Engelhart, L., Ranstam, J., Anderson, A. F., Irrgang, J., Marx, R. G., et al., ICRS recommendation document: patient reported outcome instruments for use in patients with articular cartilage defects. Cartilage. 2011;2: 122-136.

16. Eftekharsadat, B., Babaei-Ghazani, A., Habibzadeh, A., Kolahi, B. Efficacy of action potential simulation and interferential therapy in the rehabilitation of patients with knee osteoarthritis. Therapeutic advances in musculoskeletal disease. 2015 Jun; 7(3): 67-75.

17. Smith, M., Fryer, G. A comparison of two muscle energy techniques for increasing the flexibility of hamstring muscle group. J Bodyw Mov Ther. Oct 2008; 12(4): 312-317.

18. Centers for Disease Control and Prevention. Sorting Through the Evidence for the Arthritis Self-Management Program and the Chronic Disease Self-Management Program: Executive Summary of ASMP/CDSMP Meta-Analyses. May 2011. Accessed at www.cdc.gov/arthritis/docs/ASMPexecutivesummary.pdf on October 26, 2012.

19. Bjordal, J. M., Johnson, M. I., Lopes-Martins, R. A., Bogen, B., Chow, R., Ljunggren, A. E., et al., Short-term efficacy of physical interventions in osteoarthritic knee pain. A systematic review and meta-analysis of randomised placebocontrolled trials. BMC musculoskeletal disorders. 2007 Dec; 8(1): 1-14.

20. Khuman, P. R., Surbala, L., Patel, P., Chavda, D. Immediate effects of single session post isometric relaxation muscle energy technique versus mulligan's bent leg raise technique on pain and hamstring flexibility in knee osteoarthritis participants: A randomised controlled study. Physiotherapy. 2014 Sep; 3(9): 324-335.

21. Hoeksma, H. L., Dekker, J., Ronday, H. K., Heering, A., Van Der Lubbe, N., Vel, C., et al., Comparison of manual therapy and exercise therapy in osteoarthritis of the hip: a randomized clinical trial. Arthritis Care \& Research: Official Journal of the American College of Rheumatology. 2004 Oct 15; 51(5): 722-729.

22. Kumar, P., Moitra, M. Efficacy of Muscle energy technique and PNF stretching compared to conventional physiotherapy in program of Hamstring flexibility in chronic nonspecific low back pain, Ind J Physioth \& Occupa Ther. 2015 Jul-Sep; 9(3): 103-107.

23. Messier, S. P., Mihalko, S. L., Beavers, D. P., Nicklas, B. J, DeVita, P., Carr, J. J. et al., Strength Training for Arthritis Trial (START): design and rationale. BMC musculoskeletal disorders. 2013 Dec; 14(1): 1-15.

24. Zeni, J.A., Rudolph, K., Higginson, J.S. Alterations in quadriceps and hamstrings coordination in persons with medial compartment knee osteoarthritis J. Electromyogr Kinesiol. 2010; 20: 148-154. 